\title{
Perceptual properties of feedback stimuli influence the feedback-related negativity in the flanker gambling task
}

\author{
YANNI LIU, ${ }^{\mathrm{a}}$ LINDSAY D. NELSON, ${ }^{\mathrm{b}}$ EDWARD M. BERNAT, ${ }^{\mathrm{c}}$ AND WILLIAM J. GEHRING ${ }^{\mathrm{d}}$ \\ ${ }^{a}$ Department of Psychiatry, University of Michigan, Ann Arbor, Michigan, USA \\ ${ }^{b}$ Department of Neurology, Medical College of Wisconsin, Milwaukee, Wisconsin, USA \\ 'Department of Psychology, University of Maryland, College Park, Maryland, USA \\ ${ }^{\mathrm{d}}$ Department of Psychology, University of Michigan, Ann Arbor, Michigan, USA
}

\begin{abstract}
A negative deflection in the event-related potential is enhanced following error- and loss-related feedback in decisionmaking and simple gambling tasks. Researchers have assumed that the perceptual properties of the feedback stimuli are unimportant in explaining these effects. This assumption was tested in the present study through a flanker gambling task, in which the perceptual properties of the feedback were manipulated. Consistent with previous studies, loss elicited a larger feedback-related negativity (FRN) than gain feedback. However, this FRN reward effect was modulated by the perceptual properties of the feedback stimuli. When gain and loss feedback were perceptually similar to each other, the enhancement of the FRN following the loss feedback was smaller compared to when the gain and loss feedback were different from each other. In addition, incongruent feedback elicited a larger FRN than congruent feedback; this FRN congruency effect was larger following gain than loss feedback. These results suggested that perceptual properties of the feedback stimuli play a role in the elicitation of the FRN.
\end{abstract}

Descriptors: Feedback-related negativity, Flanker task, Perceptual mismatch, ERP

The feedback-related negativity (FRN) is an event-related potential (ERP) component elicited following a negative feedback stimulus indicating an incorrect choice or judgment. The FRN is maximal over medial frontal scalp locations, peaks at about 250 to $350 \mathrm{~ms}$ after the onset of the feedback stimuli, and may reflect a degree of theta phase consistency and power enhancement over the medial frontal cortex (Bernat, Nelson, Holroyd, Gehring, \& Patrick, 2008; Cavanagh, Cohen, \& Allen, 2009). Source localization analyses suggested the anterior cingulate cortex as a likely generator (Miltner et al., 2003; Ridderinkhof, Ullsperger, Crone, \& Nieuwenhuis, 2004), although this issue is not settled (Dehaene, Posner, \& Tucker, 1994; Nachev, Kennard, \& Husain, 2008; Ridderinkhof et al., 2004; Vidal, Hasbroucq, Grapperon, \& Bonnet, 2000; see Gehring, Liu, Orr, \& Carp, 2012).

The FRN was first observed by Miltner, Braun, and Coles (1997) in a time-estimation task, in which subjects had to estimate the passage of $1 \mathrm{~s}$ by pressing a button, after which they received feedback about the accuracy of the estimate. Miltner and colleagues (1997) originally proposed that the FRN reflected the operation of an error-processing system, similar to the error-related negativity (ERN) elicited upon error commission in speeded choice reaction time tasks (Falkenstein, Hohnsbein, Hoormann, \& Blanke, 1991; Gehring, Goss, Coles, Meyer, \& Donchin, 1993). Later, the component was also observed in gambling tasks when subjects made a choice and then received loss rather than gain feedback

Address correspondence to: Yanni Liu, Ph.D., 4250 Plymouth Road, Ann Arbor, MI 48109-5766, USA. E-mail: yanniliu@umich.edu about their choice (Gehring \& Willoughby, 2002; Hajcak, Holroyd, Moser, \& Simons, 2005; Yeung, Holroyd, \& Cohen, 2005; Yeung $\&$ Sanfey, 2004). Holroyd and Coles (2002) proposed the reinforcement-learning theory of the ERN (RL-ERN) to incorporate both the FRN and the response-locked ERN into a unified theoretical framework, and suggested that these components are representative of a temporal-difference error signal that is used to adjust the cognitive control system. In particular, the FRN represents an ERN elicited upon the detection of unexpected and unfavorable outcomes when feedback is given.

Consistent with the prediction of the RL-ERN theory, several studies have shown that (a) the FRN reflects neural reward prediction errors and is larger for unexpected than for expected outcomes (Hajcak, Moser, Holroyd, \& Simons, 2007; Holroyd, Krigolson, Baker, Lee, \& Gibson, 2009; Nieuwenhuis et al., 2002); (b) the FRN reflects an evaluation of events along a general good-bad dimension, and it is larger for unfavorable than for favorable outcomes (Holroyd \& Coles, 2002); and (c) the evaluation system that produces the FRN is nonlinear, weighting the worst and middle outcomes equally (Hajcak, Moser, Holroyd, \& Simons, 2006; Holroyd, Hajcak, \& Larsen, 2006; Holroyd, Larsen, \& Cohen, 2004; Yeung \& Sanfey, 2004). More recently, however, studies have questioned whether the FRN is restricted to unexpected, unfavorable outcomes, and theories have emerged suggesting that the FRN is elicited by unlikely outcomes, regardless of their valence (Ferdinand, Mecklinger, Kray, \& Gehring, 2012; Oliveira, McDonald, \& Goodman, 2007). Ferdinand et al. (2012), for example, showed that FRN activity was elicited by unexpected, 
positive response outcomes in a time-estimation task. They argued that the results supported an alternative to the RL-ERN theory, the predicted response outcome (PRO) model of Alexander \& Brown (2010, 2011), according to which the anterior cingulate is sensitive to unexpected response outcomes, regardless of their valence.

In previous studies, researchers have often assumed that the FRN is unaffected by the perceptual properties of feedback stimuli (Hajcak et al., 2006; Holroyd \& Coles, 2002; Holroyd, Nieuwenhuis, Yeung, \& Cohen, 2003; Miltner et al., 1997). However, there is evidence against the assumption. A feedback stimulus can signify whether a response was associated with a gain or a loss, whether it was correct or erroneous, or it can convey both pieces of information (such as when a response is erroneous by virtue of yielding a gain that is smaller than desired). Nieuwenhuis, Yeung, Holroyd, Schurger, and Cohen (2004) showed that the FRN responded to the most perceptually salient information conveyed by a feedback stimulus and was not responsive to the same information when the information was not perceptually salient. The FRN can also be determined simply by the perceptual discrepancy between two consecutive feedback stimuli. Using a guessing task, where participants had to decide whether the first stimulus would have the same color as the second stimulus that was subsequently presented, Jia et al. (2007) showed that the FRN effect was not only determined by the feedback concerning the correctness of one's performance, but also by the perceptual discrepancy between the first stimulus and the second stimulus. In a previous study, in which a single visual feature or a conjunction of features was used to indicate reward feedback, we found that losses elicited a larger FRN than gain feedback only in the single-feature condition but not in the conjoined-feature condition, suggesting that the FRN is modulated by the deviation of feedback stimuli from a perceptual template (Liu \& Gehring, 2009).

Such findings are not necessarily inconsistent with either the RL-ERN theory or the PRO model, but rather point to a need for a greater clarity about the representations underlying the computations in those models. For example, the RL-ERN theory is vague about the representation in the system that generates the FRN-it does not say whether the perceptual attributes of feedback stimuli will or will not affect the FRN. Does the basal ganglia comparator at the heart of the RL-ERN model depend on early automatic perceptual processing, later more attention-dependent processing, or both? In short, greater specificity is needed about what exactly is expected and what is unexpected in these models.

The current study is an effort to clarify the empirical basis for these models. Although there are many ways that perceptual representations could be used as the basis for feedback processing, one general question is whether the computation underlying the FRN requires focused attention or whether the processing can proceed on the basis of preattentive feature analysis (Treisman \& Gelade, 1980). The findings of Liu and Gehring (2009) described earlier support the latter interpretation. Here, we explored another implication of this interpretation: that the FRN will be sensitive to irrelevant attributes of a feedback stimulus that mismatch perceptually the expected or desired gain stimulus. The two irrelevant attributes we explored were (1) the presence of irrelevant stimuli flanking the actual feedback stimulus, and (2) the visual similarity of the gain and loss feedback stimuli. Neither attribute had an effect on the amount actually gained or lost by the subject, and our question was whether these attributes would affect the FRN, thus dissociating the FRN responses from the actual monetary outcome.

In our study, we used a gambling task in which participants chose one of four doors presented on the screen and then received gain or loss feedback about each choice. Feedback stimuli comprised perceptual attributes that conveyed the gain or loss outcome, as well as irrelevant perceptual attributes. We manipulated the congruence of the irrelevant perceptual information in the stimulus: the feedback information was indicated by a central letter in a congruent or incongruent five-letter string often used in the classic Eriksen flanker letter task (Eriksen \& Eriksen, 1974). In the congruent letter string, the center target letter and its surrounding flanker letters are identical (e.g., HHHHH); in the incongruent letter string, the center target letter and its surrounding flanker letters are different (e.g., SSHSS). The flanking letters did not convey any actual gain or loss reward information.

We also manipulated the perceptual similarity of letters indicating gain and loss. Based on the hypothesis that the process responsible for the FRN was sensitive to preattentive feature analysis, it was predicted that dissimilar letters (e.g., letter $\mathrm{S}$ and $\mathrm{T}$ indicated gain and loss, respectively) would elicit a larger FRN than similar letters (e.g., letter $\mathrm{E}$ and $\mathrm{F}$ indicated gain and loss, respectively) due to a larger perceptual mismatch between the actual outcome and the expected mental template. For each set of letters, there were four types of flanker letter strings: congruent gain, where all five letters indicated gain (e.g., SSSSS); congruent loss, where all five letters indicated loss (e.g., TTTTT); incongruent gain, where the middle target letter indicated gain, surrounded by four letters indicating loss (e.g., TTSTT); and incongruent loss, where the middle target letter indicated loss, surrounded by four letters indicating gain (e.g., SSTSS). It was predicted that the incongruent gain feedback may elicit a larger FRN than congruent gain feedback for two reasons: (1) the increased mismatch between the expected gain-related features and the presence of the loss-related perceptual features in the flanker letters, and (2) the increased mismatch between the gain target and the loss flankers embedded in the feedback stimulus itself (as in the classic Eriksen flanker letter task). Strictly speaking, any theory such as the RL-ERN theory that links the FRN only to the actual gain or loss delivered by a feedback stimulus would not predict an enhanced FRN for the incongruent gain, because the stimulus would still be categorized as a favorable outcome.

\section{Method}

\section{Participants}

There were 12 participants ( 6 males and 6 females) aged between 18 and 23 years old. All were right-handed, had normal or corrected-to-normal vision, and normal color vision. Prior to the test, participants provided written informed consent in accordance with the Institutional Review Board of the University of Michigan. They received a monetary payment for their participation.

\section{Procedure}

The participants were seated comfortably $60 \mathrm{~cm}$ in front of a 14" CRT computer monitor in a dimly lit, sound-attenuating, and electromagnetically shielded room. They were instructed to remain as still as possible and to minimize eye blinks throughout the experiment. Materials were presented using E-Prime (Psychological Software Tools, Pittsburgh, PA). On each trial of the experiment, the participants were presented with four identical red doors displayed at the center of the screen following a 500-ms central fixation, and were instructed that the reward information was hidden behind those doors (Figure 1). Doors remained on the 


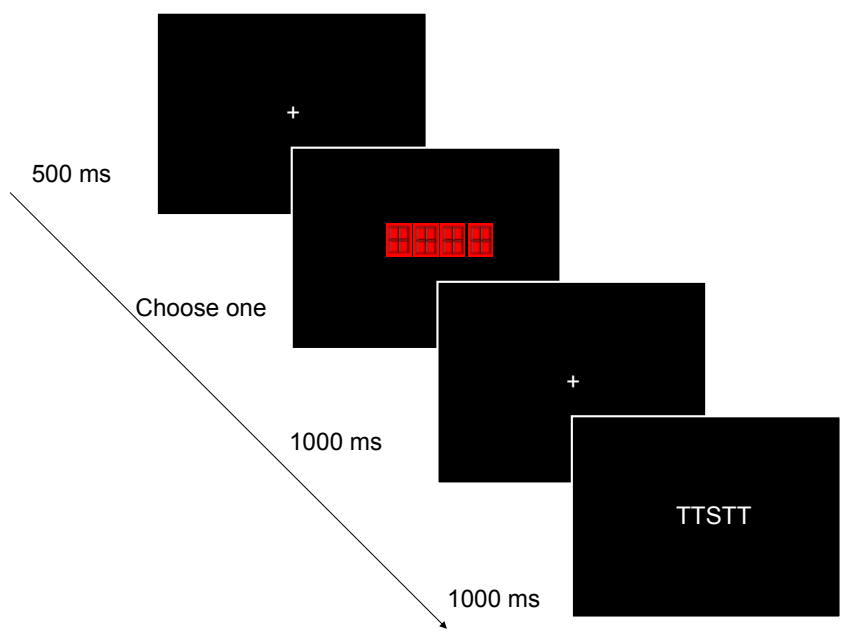

Figure 1. Experimental paradigm.

screen until the participants selected one by pressing a button with their left or right index or middle finger, corresponding to the location of the chosen door. One thousand milliseconds after the response, the reward information indicating a gain or loss on the trial appeared for $1,000 \mathrm{~ms}$. The intertrial interval was $1,000 \mathrm{~ms}$.

The reward information was a letter string in the form of the flanker stimuli-one target letter (e.g., letter S) at the center was surrounded by identical (e.g., SSSSS; congruent condition) or different letters (e.g., TTSTT; incongruent condition). Only the central letter conveyed the reward information. The perceptual similarity of the gain and loss letters was manipulated. In half of the trials, gain and loss information was indicated by two perceptually similar letters: E versus F; in the other half of the trials, gain and loss information was indicated by two perceptually dissimilar letters: S versus T. Participants completed five blocks of EF feedback (similar blocks) and five blocks of ST feedback (dissimilar blocks). The presentation sequence of the two types of blocks was counterbalanced among the participants. Each block started with 50 cents as the initial allotment, and on each trial participants won or lost 25 cents. There were 120 trials per block, and after every 12 trials, participants were given summary information on the computer display about the bonus they had earned. The gain or loss information each letter indicated was revealed to the participants at the beginning of the experiment and was counterbalanced among the participants. The feedback was randomly chosen from a set of equal numbers of congruent and incongruent as well as gain and loss feedback. The number of trials of each letter string (similar blocks: EEEEE, FFEFF, FFFFF, EEFEE; dissimilar blocks: SSSSS, SSTSS, TTTTT, TTSTT) was about the same, the percentage of gain or loss was approximately $50 \%$, and the ratio of congruent to incongruent trials was roughly $1: 1$. Before the experiment, the participants did the practice block with 12 trials, and the experimenter confirmed that participants understood the task.

\section{Electrophysiological Methods}

The electroencephalogram (EEG) was recorded from 26 scalp electrodes with $\mathrm{Ag} / \mathrm{AgCl}$ electrodes embedded in a nylon mesh cap (Easy-Cap, Falk Minow Systems, Inc.). The electrode locations consisted of FP1, AFz, FP2, F7, F3, Fz, F4, F8, FC3, FCz, FC4, T7, C3, Cz, C4, T8, CP3, CPz, CP4, P7, P3, Pz, P4, P8, O1, and O2.
EEG data were recorded with a left mastoid reference and a forehead ground. An average mastoid reference was derived offline using both left and right mastoid data. The vertical electrooculogram (EOG) was recorded from two $\mathrm{Ag} / \mathrm{AgCl}$ electrodes placed respectively above and below the left eye; the horizontal EOG was recorded from electrodes placed external to the outer canthus of each eye. Impedances were kept below $10 \mathrm{~K} \Omega$. EEG and EOG were amplified by SYNAMPS DC amplifiers (Neuroscan Labs, Sterling, VA, USA) and filtered online from .01 to $100 \mathrm{~Hz}$ (halfamplitude cutoffs). The data were digitized at $500 \mathrm{~Hz}$.

EEG epochs of $1,100 \mathrm{~ms}$ (100-ms baseline) were extracted offline from the continuous data file for analysis. Data were screened automatically to eliminate epochs that contained unvarying amplitude values for more than $20 \mathrm{~ms}$, but no such epochs were identified. Ocular artifacts were corrected using the algorithm described by Gratton, Coles, and Donchin (1983). Statistical analyses were performed on the data without any additional filtering. The data presented in the figures were filtered with a nine-point Chebyshev II low-pass digital filter with a half-amplitude cutoff at $12 \mathrm{~Hz}$ (MATLAB 7.04; Mathworks Inc.).

In addition to the measurement in the time domain, we computed a time-frequency (TF) counterpart to the time-domain FRN in order to differentiate it from potential overlapping components at other frequencies (e.g., especially the P300, which overlaps with the FRN in time but is dominated by activity in a lower frequency band and therefore is separable by TF analysis; Bernat, Nelson, Steele, Gehring, \& Patrick, 2011; Nelson, Patrick, Collins, Lang, \& Bernat, 2011). This entailed first computing TF energy distribution (surface) of the time-domain ERP using the binomial reduced interference distribution variant of Cohen's class of time-frequency transforms (for details, see Bernat, Williams, \& Gehring, 2005). Next, TF principal components analysis (TF-PCA) was applied to an area corresponding to the $0-750 \mathrm{~ms}$ time range and $3.5-6.5 \mathrm{~Hz}$ frequency range (based on prior work demonstrating that FRN activity occurs primarily in this theta frequency band; e.g., Bernat et al., 2011; Gehring \& Willoughby, 2004; Nelson et al., 2011). Five components were extracted based on visual inspection of the scree plot, and one was selected for analysis as it best matched the temporal and spatial characteristics of the time-domain FRN (e.g., it was maximal around the same latency as the time-domain FRN, it showed enhancement for loss vs. gain trials at frontocentral scalp sites, and the loss-gain difference score correlated more strongly than other PCs with the FRN loss-gain difference, $r=.84$ ). Electrode $\mathrm{FCz}$ was most proximal topographically to the maximum theta gain-loss condition difference, and hence data from this electrode was used in the statistical analyses of theta-FRN.

\section{Results}

The FRN is characterized by the negative deflection that peaked about $300 \mathrm{~ms}$ following the feedback. The FRN mean amplitude between $250 \mathrm{~ms}$ and $350 \mathrm{~ms}$ following the feedback was measured at $\mathrm{FCz}$ (see Table 1). A $2 \times 2 \times 2$ three-way repeated measures analysis of variance (ANOVA) with factors block type (similar vs. dissimilar), reward valence (gain vs. loss), and congruency (congruent vs. incongruent) revealed main effects of reward valence, $F(1,11)=19.31, p<.01, \eta_{\mathrm{p}}^{2}=.637$, and congruency $F(1,11)=$ $9.83, p<.01, \eta_{\mathrm{p}}^{2}=.472$, in addition to interactions between reward valence and congruency, $F(1,11)=8.11, p<.05, \eta_{\mathrm{p}}^{2}=.424$, and between reward valence and block type, $F(1,11)=5.10, p<.05$, $\eta_{\mathrm{p}}^{2}=.317$. There were no other main effects or interaction effects. Overall, loss feedback elicited a larger FRN than gain feedback, 
Table 1. Descriptive Statistics of Mean Amplitude, Baseline-to-Peak Amplitude, and Peak Latency for Each Condition

\begin{tabular}{llccc}
\hline \hline & & $\begin{array}{c}\text { Mean amplitude }(\mu \mathrm{V}) \\
(\text { mean } \pm S D)\end{array}$ & $\begin{array}{c}\text { Peak amplitude }(\mu \mathrm{V}) \\
(\text { mean } \pm S D)\end{array}$ & $\begin{array}{c}\text { Peak latency }(\mathrm{ms}) \\
(\mathrm{mean} \pm S D)\end{array}$ \\
\hline Similar & & $7.9 \pm 5.2$ & $5.0 \pm 4.5$ & $282.5 \pm 41.0$ \\
& Gain_congurent & $6.3 \pm 4.0$ & $3.7 \pm 4.2$ & $311.3 \pm 47.5$ \\
& Gain_incongruent & $6.2 \pm 4.8$ & $3.4 \pm 4.8$ & $299.5 \pm 53.0$ \\
& Loss_congruent & $5.8 \pm 4.1$ & $3.6 \pm 4.2$ & $321.8 \pm 55.0$ \\
Dissimilar & Loss_incongruent & $9.2 \pm 5.4$ & $6.4 \pm 5.2$ & $282.8 \pm 62.0$ \\
& Gain_congruent & $6.9 \pm 4.6$ & $3.9 \pm 4.2$ & $300.5 \pm 37.4$ \\
& Gain_incongruent & $6.2 \pm 4.6$ & $2.2 \pm 2.8$ & $303.5 \pm 48.9$ \\
& Loss_congruent & $5.2 \pm 2.9$ & & $323.7 \pm 41.7$ \\
\hline \hline
\end{tabular}

Note. $S D=$ standard deviation.

and incongruent feedback elicited a larger FRN than congruent feedback. Consistent with our hypotheses, the congruency effect was evident only in the FRN elicited by gain feedback, $F(1,11)=14.44, p<.01, \eta_{\mathrm{p}}^{2}=.568$, but not in the FRN elicited by loss feedback, $F(1,11)=2.57, p>.10, \eta_{\mathrm{p}}^{2}=.189$. The reward effect was evident in both similar, $F(1,11)=11.24, p<.01, \eta_{\mathrm{p}}^{2}=.505$, and dissimilar, $F(1,11)=15.61, p<.01, \eta_{\mathrm{p}}^{2}=.587$, blocks, but the effect was larger in the dissimilar blocks than that in the similar blocks (Block Type $\times$ Valence interaction, $F(1,11)=5.10, p<.05$, $\left.\eta_{\mathrm{p}}^{2}=.317\right)$.

Inspection of the ERP waveforms in Figure 2 suggested that the mean amplitude analyses using $250-350 \mathrm{~ms}$ as the time window would not catch the late enhancement of the FRN in the incongruent-loss feedback condition, so a baseline-to-peak measurement was conducted at $\mathrm{FCz}$ as well. The peak amplitude of the FRN was defined as the most negative value of the ERP waveforms between $200 \mathrm{~ms}$ and $400 \mathrm{~ms}$ following the feedback (relative to the prestimulus baseline), and the peak latency of the FRN was defined as the time when the most negative peak occurred between $200 \mathrm{~ms}$ and $400 \mathrm{~ms}$ (see Table 1). Consistent with the mean amplitude analyses and our hypotheses, the reward effect was significant in the dissimilar blocks, $F(1,11)=10.28, p<.01, \eta_{\mathrm{p}}^{2}=.483$, and marginally significant in the similar blocks, $F(1,11)=4.31, p=.06$, $\eta_{\mathrm{p}}^{2}=.281$. The Block Type $\times$ Valence interaction revealed a trend toward the reward effect being larger in the dissimilar block than in the similar block, $F(1,11)=4.66, p=.054, \eta_{\mathrm{p}}^{2}=.298$. Meanwhile, the congruency effect was evident for gain feedback, $F(1,11)=19.60, p<.01, \eta_{\mathrm{p}}^{2}=.641$, but not for loss feedback, $F(1,11)=1.70, p>.10, \eta_{\mathrm{p}}^{2}=.134$. The congruency effect in the gain feedback appears to be larger than that in the loss feedback (Congruency $\times$ Valence interaction, $F(1,11)=4.65, \quad p=.054$, $\left.\eta_{\mathrm{p}}^{2}=.297\right)$. For the peak latency, the FRN elicited by incongruent feedback was longer than that by congruent feedback, $F(1,11)=$ $15.88, p<.01, \eta_{\mathrm{p}}^{2}=.591$. There were no main effects of block type, $F<1, \eta_{\mathrm{p}}^{2}=.002$, and valence, $F(1,11)=3.04, p>.10, \eta_{\mathrm{p}}^{2}=.216$, or any interactions $(F \mathrm{~s}<1)$ in peak latency analyses.

Visual inspection of the ERP waveform suggested that the FRN may have overlapped lower-frequency (P300) activity, so a timefrequency theta-FRN component was extracted using TF-PCA in order to isolate theta-FRN from this extraneous lower frequency activity (see Method for details, Figure 3). Figure 3 showed that the theta measure is squarely over the FRN and does not overlap with P300. Consistent with the measurements in the time domain, loss feedback increased the theta-FRN relative to gain feedback, $F(1,11)=10.92, p<.01, \eta_{\mathrm{p}}^{2}=.498$. The reward effect was significant in dissimilar blocks, $F(1,11)=12.96, p<.01, \eta_{\mathrm{p}}^{2}=.541$, and only marginally significant in similar blocks, $F(1,11)=4.68$, $p=.053, \eta_{\mathrm{p}}^{2}=.298$. The Block Type $\times$ Valence interaction revealed a trend toward the reward effect being larger in the dissimilar block than in the similar block, $F(1,11)=4.80, p=.051, \eta_{\mathrm{p}}^{2}=.304$. The
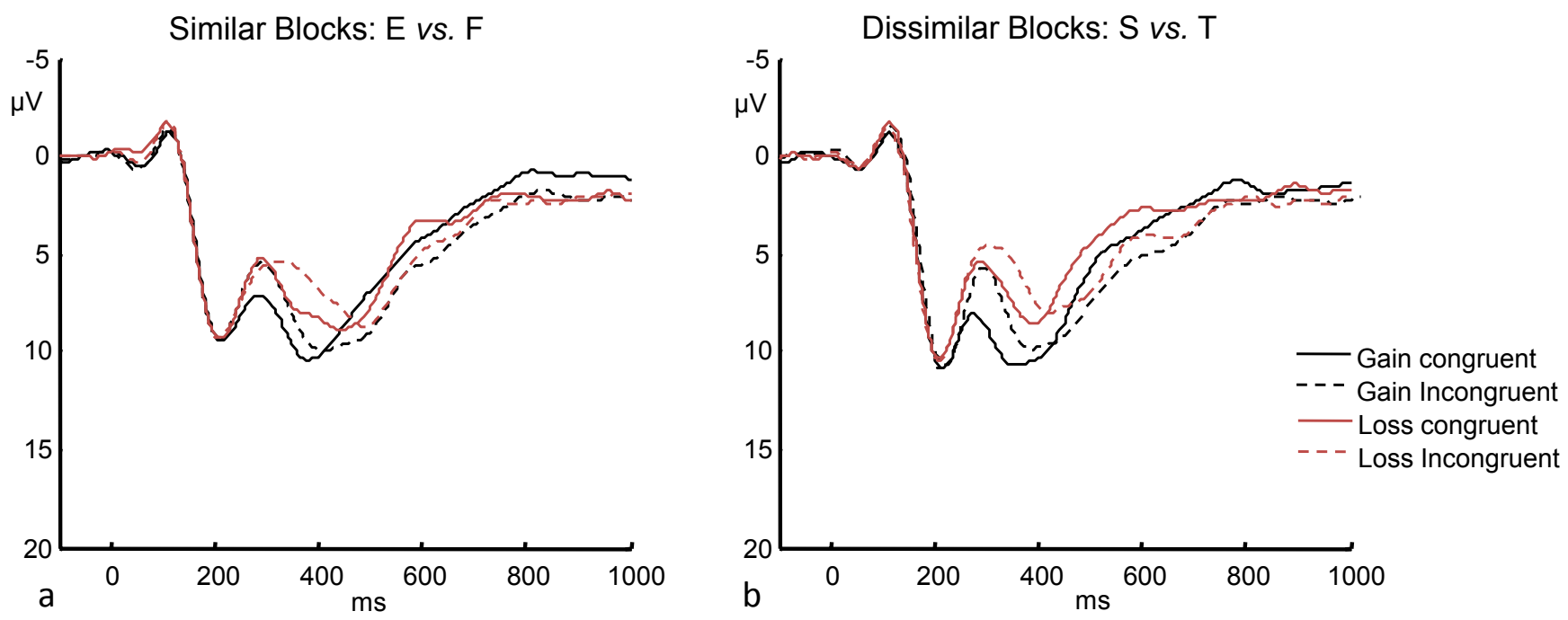

Figure 2. Grand average of ERP recordings at FCz for (a) similar and (b) dissimilar blocks. Feedback stimuli appear at 0 ms. 

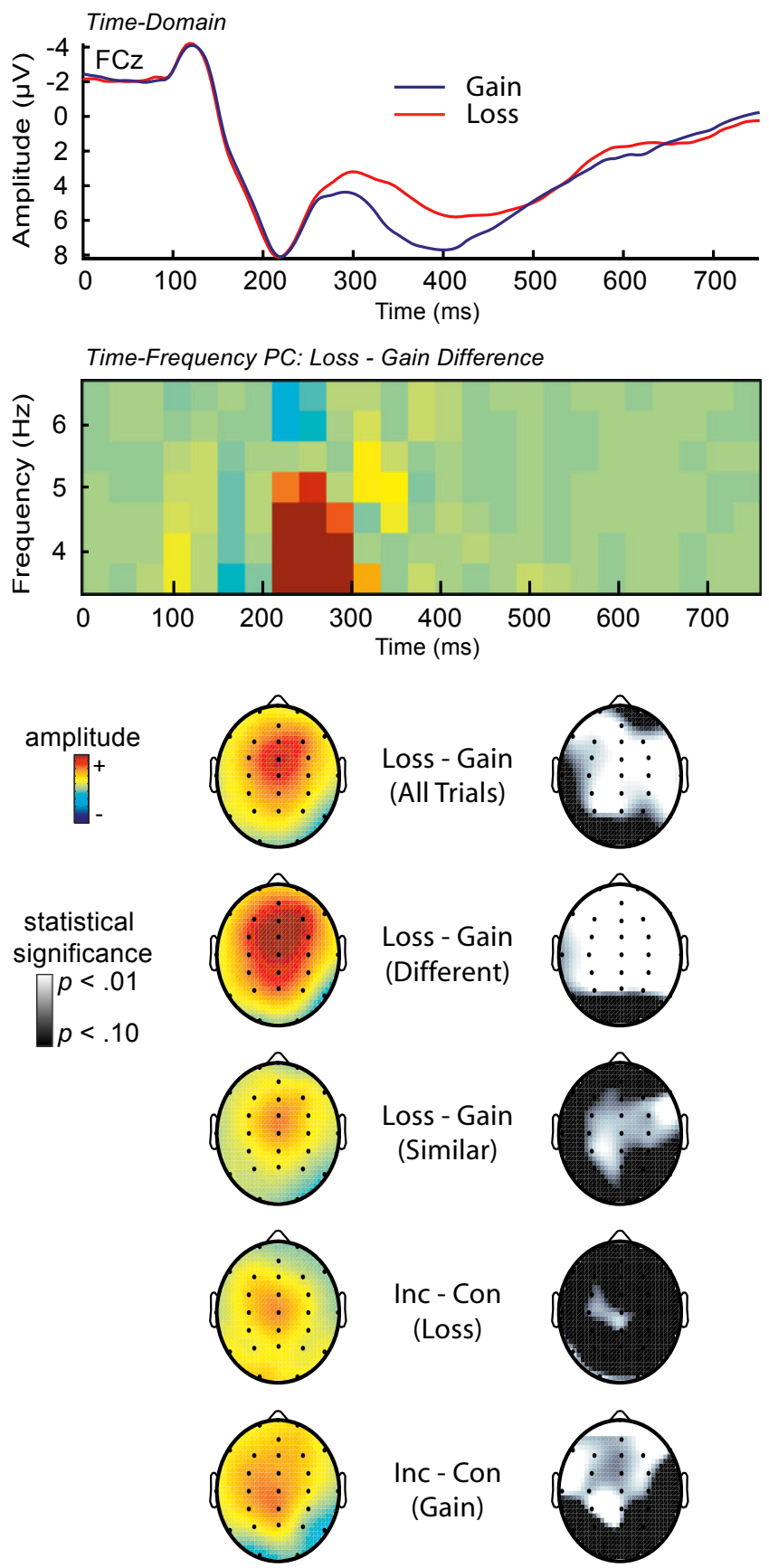

Figure 3. Time-frequency principal components decomposition of event-related potential activity. Top row: time-domain waveform plot at $\mathrm{FCz}$, depicting the expected amplitude difference for loss vs. gain trials in the feedback-related negativity (FRN). Second row: Time-frequency surface for primary activity in the theta $(3.5-6.5 \mathrm{~Hz})$ range corresponding to FRN, depicted for loss (red) versus gain (blue) trials at FCz (green corresponds to areas with no difference between loss and gain trials, as depicted in the amplitude color legend). Bottom rows: Topographic maps depict important condition differences in terms of overall magnitude (left; color surface plots) and statistical significance (right; using Wilcoxon $p$ values for illustration of strength and topography of effects). $\mathrm{PC}=$ principal component. incongruent gain feedback increased the theta-FRN relative to congruent gain feedback, $F(1,11)=5.17, p<.05, \eta_{\mathrm{p}}^{2}=.320$; there was no difference between incongruent loss feedback and congruent loss feedback, $F(1,11)=2.43, p=.15, \eta_{\mathrm{p}}^{2}=.181$. However, the interaction between congruency and valence was not significant $\left(F<1, \eta_{\mathrm{p}}^{2}=0\right)$.

\section{Discussion}

In the present study, we examined how the perceptual properties of feedback stimuli may modulate the FRN by manipulating the reward valence, congruency, and perceptual similarity of the feedback stimuli in a gambling task where flanker stimuli were used. Consistent with previous findings (Gehring \& Willoughby, 2002; Holroyd \& Coles, 2002), loss feedback elicited a larger FRN than gain feedback, and this FRN reward effect was larger in dissimilarletter than similar-letter feedback. In addition, incongruent letter strings elicited a larger FRN than congruent letter strings, and this FRN congruency effect was larger for gain feedback than for loss feedback.

\section{FRN Reward Effect}

The increased FRN reward effect in dissimilar blocks compared to similar blocks was consistent with the proposal that the FRN is affected by the perceptual mismatch between gain and loss feedback stimuli (Folstein \& van Petten, 2008; Liu \& Gehring, 2009). In the gambling task, one way in which such an effect could occur would be tuning (or priming) in the perceptual system for the perceptual properties of gain-related feedback; any feedback outcome that deviates from the prepared gain-related perceptual representation would trigger the monitoring system to elicit an error signal. The larger perceptual mismatch between loss and gain feedback in the dissimilar-letter condition may generate a larger error signal than in the similar-letter feedback condition, resulting in a larger FRN. Results of time-frequency analysis further confirmed that the interaction was not due to ERP components of different frequency (e.g., P300) overlap in time but truly reflected the FRN occurring at around 3.5 to $6.5 \mathrm{~Hz}$ (Bernat et al., 2011; Gehring \& Willoughby, 2004).

The findings in the current study are consistent with the results in our previous study (Liu \& Gehring, 2009), where the FRN reward effect was found in the single-feature condition but not in the conjoined-feature condition. Both studies suggested that the FRN reward effect was sensitive to the preattentive features of the feedback stimuli (Treisman \& Gelade, 1980). Models applicable to the FRN (e.g., Alexander \& Brown, 2011; Holroyd \& Coles, 2002) should incorporate computational processes making clear how the preattentive feature analysis of the feedback stimuli affects the representation underlying the FRN computation. Furthermore, a reduced FRN reward effect was found in the similar-letter condition in the current study, where attention is needed to discriminate loss from gain feedback stimuli, suggesting that attentiondependent processing may also be present in the computation of the FRN. More studies are needed to address the role of early automatic perceptual processing and later attentional processing in the FRN computations.

Of note, the interaction between block type and reward valence was significant in the FRN mean amplitude measure, and only marginally significant in the FRN peak amplitude and timefrequency theta-FRN measures $(p s<.06)$. The partial eta squared (i.e., $\eta_{\mathrm{p}}^{2}$ ) effect size measures showed a large effect size corre- 
sponding to the interaction between block type and reward valence-0.317, 0.297, and 0.298, respectively, for FRN mean amplitude, peak amplitude, and theta-FRN measures (Brown, 2008). The fact that these results occur after our stringent efforts to account for possible contamination from overlapping ERP components lends increased confidence to the finding in mean amplitude measures of an increased FRN reward effect in dissimilar-letter condition compared to similar-letter condition. It is possible that larger differences would be observed if the perceptual difference between the dissimilar and similar conditions were increased. Finally, we should note that, without behavioral measures directly reflecting the gain/loss discrimination, it is difficult to rule out stimulus equivocation as a possible contributor to the observed FRN differences. Incorporating additional behavioral measures to indicate that stimuli were perceived correctly as gains and losses would be desirable.

\section{FRN Congruency Effect}

In addition to the reward effect, the FRN elicited by incongruent letter strings was larger than that elicited by congruent letter strings, especially those elicited by gain feedback stimuli. The FRN congruency effect was similar to the $\mathrm{N} 2$ effect observed in the classic Eriksen flanker task, in which incongruent stimuli elicit a central or frontocentral N2. As reviewed in Folstein and van Petten (2008), both response conflict and the perceptual mismatch between central target and surrounded flanker letters may contribute to the frontal N2 in the classic flanker task. In contrast to the classic flanker task, the flankers in the current gambling task had no response-related features. In addition to the perceptual mismatch between central and flanker letters, the FRN may be sensitive to the mismatch between the reward information conveyed by central letters and that conveyed by flanker letters, which was not dissociated from perceptual mismatch in the current experimental design.
Another potential factor contributing to the FRN enhancement in the incongruent gain condition is the increased mismatch between the expected gain-related features and the presence of the lossrelated perceptual features in the flanker letters. If the perceptual system is tuned for the perceptual properties of gain-related feedback, the presence of loss-related perceptual features in incongruent-flanker gain feedback deviates from the prepared gainrelated perceptual representation, which triggers an error signal. In contrast, in the incongruent flanker loss condition, there is no mismatch between the expected gain-related features and the gainrelated perceptual features in the flanker letters; instead, the appearance of gain-related features in the incongruent-flanker loss condition would reduce the FRN congruency effect elicited by mismatch between central and flanker letters. Thus, the congruency effect in the loss condition is smaller than that in the gain condition.

Recent theorizing suggested that an enhanced positivity (e.g., a frontal P2) in the gain feedback may be responsible for the FRN or may overlap it (Holroyd \& Coles, 2008; Potts, Martin, Burton, \& Montague, 2006). Visual inspection of our waveforms suggested that a P2 difference cannot account for our results, with our FRN findings primarily occurring after the $\mathrm{P} 2$. We cannot, however, rule out contributions from a positivity that perfectly overlaps in time with the negative peak of the FRN.

In summary, we found that the FRN amplitude was modulated by the perceptual similarity between gain and loss feedback stimuli and was enhanced by the congruence of perceptual features in the stimuli. Our findings suggest that theories of the FRN must address the ways that feedback-related processing interacts with perceptual processing of stimulus attributes. In particular, the present data point toward preattentive inputs to the neural processing reflected in the FRN. At a more practical level, the findings suggest that researchers must control the perceptual properties of the feedback stimuli when using the FRN to investigate decision-making or reward-related processing.

\section{References}

Alexander, W. H., \& Brown, J. W. (2010). Computational models of performance monitoring and cognitive control. Topics in Cognitive Science, 2, 658-677. doi: 10.1111/j.1756-8765.2010.01085.x

Alexander, W. H., \& Brown, J. W. (2011). Medial prefrontal cortex as an action outcome predictor. Nature Neuroscience, 14, 1338-1344. doi: $10.1038 / \mathrm{nn} .2921$

Bernat, E. M., Nelson, L. D., Holroyd, C. B., Gehring, W. J., \& Patrick, C. J. (2008). Separating cognitive processes with principal components analysis of EEG time-frequency distributions. Proceedings of the Society of Photo-Optical Instrumentation Engineers, 7074, 70740S.

Bernat, E. M., Nelson, L. D., Steele, V. R., Gehring, W. J., \& Patrick, C. J. (2011). Externalizing psychopathology and gain/loss feedback in a simulated gambling task: Dissociable components of brain response revealed by time-frequency analysis. Journal of Abnormal Psychology, 120, 352-364. doi: 10.1037/a0022124

Bernat, E. M., Williams, W. J., \& Gehring, W. J. (2005). Decomposing ERP time-frequency energy using PCA. Clinical Neurophysiology, 116, 1314-1334. doi: 10.1016/j.clinph.2005.01.019

Brown, J. D. (2008). Statistics corner. Questions and answers about language testing statistics: Effect size and eta squared. Shiken: JALT testing \& evaluation. SIG Newsletter, 12, 38-43.

Cavanagh, J. F., Cohen, M. X., \& Allen, J. J. (2009). Prelude to and resolution of an error: EEG phase synchrony reveals cognitive control dynamics during action monitoring. Journal of Neuroscience, 29, 98-105. doi: 10.1523/JNEUROSCI.4137-08.2009

Dehaene, S., Posner, M. I., \& Tucker, D. M. (1994). Localization of a neural system for error-detection and compensation. Psychological Science, 5, 303-305. doi: 10.1111/j.1467-9280
Eriksen, B. A., \& Eriksen, C. W. (1974). Effects of noise letters upon the identification of a target letter in a nonsearch task. Perception \& Psychophysics, 16, 143-149. doi: 10.3758/bf03203267

Falkenstein, M., Hohnsbein, J., Hoormann, J., \& Blanke, L. (1991). Effects of crossmodal divided attention on late ERP components. II. Error processing in choice reaction tasks. Electroencephalography and Clinical Neurophysiology, 78, 447-455. doi: 10.1016/0013-4694(91) 90062-9.

Ferdinand, N. K., Mecklinger, A., Kray, J., \& Gehring, W. J. (2012). The processing of unexpected positive response outcomes in the mediofrontal cortex. Journal of Neuroscience, 32, 12087-12092. doi: 10.1523/JNEUROSCI.1410-12.2012

Folstein, J. R., \& van Petten, C. V. (2008). Influence of cognitive control and mismatch on the N2 component of the ERP: A review. Psychophysiology, 45, 152-170. doi: 10.1111/j.1469-8986.2007. 00602.x

Gehring, W. J., Goss, B., Coles, M. G. H., Meyer, D. E., \& Donchin, E. (1993). A neural system for error detection and compensation. Psychological Science, 4, 385-390. doi: 10.1111/j.1467-9280.1993.tb00586.x

Gehring, W. J., Liu, Y., Orr, J., \& Carp, J. (2012). The error-related negativity (ERN/Ne). In S. J. Luck \& E. Kappenman (Eds.), Oxford handbook of event-related components (pp. 231-291). New York, NY: Oxford University Press.

Gehring, W. J., \& Willoughby, A. R. (2002). The medial frontal cortex and the rapid processing of monetary gains and losses. Science, 295, 22792282. doi: $10.1126 /$ science. 1066893

Gehring, W. J., \& Willoughby, A. R. (2004). Are all medial frontal negativities created equal? Toward a richer empirical basis for theories of action monitoring. In M. Ullsperger \& M. Falkenstein (Eds.), Errors, 
conflicts, and the brain. Current opinions on performance monitoring (pp. 14-20). Leipzig, Germany: Max Planck Institute of Cognitive Neuroscience.

Gratton, G., Coles, M. G. H., \& Donchin, E. (1983). A new method for off-line removal of ocular artifact. Electroencephalography and Clinical Neurophysiology, 55, 468-484. doi: 10.1016/0013-4694(83) 90135-9

Hajcak, G., Holroyd, C. B., Moser, J. S., \& Simons, R. F. (2005). Brain potentials associated with expected and unexpected good and bad outcomes. Psychophysiology, 42, 161-170. doi: 10.1111/j.1469-8986. 2005.00278.x

Hajcak, G., Moser, J. S., Holroyd, C. B., \& Simons, R. F. (2006). The feedback-related negativity reflects the binary evaluation of good versus bad outcomes. Biological Psychology, 71, 148-154. doi: 10.1016/ j.biopsycho.2005.04.001

Hajcak, G., Moser, J. S., Holroyd, C. B., \& Simons, R. F. (2007). It's worse than you thought: The feedback negativity and violations of reward prediction in gambling tasks. Psychophysiology, 44, 905-912. doi: 10.1111/j.1469-8986.2007.00567.x

Holroyd, C. B., \& Coles, M. G. H. (2002). The neural basis of human error processing: Reinforcement learning, dopamine, and the error-related negativity. Psychological Review, 109, 679-709. doi: 10.1037/ 0033295X.109.4.679

Holroyd, C. B., \& Coles, M. G. H. (2008). Dorsal anterior cingulate cortex integrates reinforcement history to guide voluntary behaviour. Cortex, 44, 548-559. doi: 10.1016/j.cortex.2007.08.013

Holroyd, C. B., Hajcak, G., \& Larsen, J. T. (2006). The good, the bad and the neutral: Electrophysiological responses to feedback stimuli. Brain Research, 1105, 93-101. doi: 10.1016/j.brainres.2005.12.015

Holroyd, C. B., Krigolson, O. E., Baker, R., Lee, S., \& Gibson, J. (2009). When is an error not a prediction error? An electrophysiological investigation. Cognitive, Affective \& Behavioral Neuroscience, 9, 59-70. doi:10.3758/CABN.9.1.59.

Holroyd, C. B., Larsen, J. T., \& Cohen, J. D. (2004). Context dependence of the event-related brain potential associated with reward and punishment. Psychophysiology, 41, 245-253. doi: 10.1111/j.1469-8986. 2004.00152.x

Holroyd, C. B., Nieuwenhuis, S., Yeung, N., \& Cohen, J. D. (2003). Errors in reward prediction are reflected in the event-related brain potential. NeuroReport, 14, 2481-2484. doi: 10.1097/01.wnr.0000099601/ 41403.a5

Jia, S., Li, H., Luo, Y., Chen, A., Wang, B., \& Zhou, X. (2007). Detecting perceptual conflict by the feedback-related negativity in brain potentials. NeuroReport, 18, 1385-1388. doi: 10.1097/WNR.0b013e $3282 \mathrm{c} 48 \mathrm{a} 90$

Liu, Y., \& Gehring, W. J. (2009). Loss feedback negativity elicited by single- versus conjoined-feature stimuli. NeuroReport, 20, 632-636. doi: 10.1097/WNR.0b013e32832a3250

Miltner, W. H., Braun, C. H., \& Coles, M. G. (1997). Event-related brain potentials following incorrect feedback in a time-estimation task: Evi- dence for a "generic" neural system for error detection. Journal of Cognitive Neuroscience, 9, 788-798. doi: 10.1162/jocn.1997.9.6.788

Miltner, W. H., Lemke, U., Weiss, T., Holroyd, C., Scheffers, M. K., \& Coles, M. G. (2003). Implementation of error-processing in the human anterior cingulate cortex: A source analysis of the magnetic equivalent of the error-related negativity. Biological Psychology, 64, 157-166. doi: 10.1016/S0301-0511(03)00107-8

Nachev, P., Kennard, C., \& Husain, M. (2008). Functional role of the supplementary and pre-supplementary motor areas. Nature Reviews Neuroscience, 9, 856-869. doi: 10.1038/nrn2478

Nelson, L. D., Patrick, C. J., Collins, P., Lang, A. R., \& Bernat, E. M. (2011). Alcohol impairs brain reactivity to explicit loss feedback in a gambling task. Psychopharmacology, 218, 419-428. doi: 10.1007/ s00213-011-2323-3

Nieuwenhuis, S., Ridderinkhof, K. R., Talsma, D., Coles, M. G., Holroyd, C. B., Kok, A., \& van der Molen, M. W. (2002). A computational account of altered error processing in older age: Dopamine and the error-related negativity. Cognitive, Affective \& Behavioral Neuroscience, 2, 19-36. doi: 10.3758/CABN.2.1.19

Nieuwenhuis, S., Yeung, N., Holroyd, C. B., Schurger, A., \& Cohen, J. D. (2004). Sensitivity of electrophysiological activity from medial frontal cortex to utilitarian and performance feedback. Cerebral Cortex, 14, 741-747. doi: 10.1093/cercor/bhh034

Oliveira, F. T., McDonald, J. J., \& Goodman. D. (2007). Performance monitoring in the anterior cingulate is not all error related: Expectancy deviation and the representation of action-outcome associations. Journal of Cognitive Neuroscience, 19, 1994-2004. doi: 10.1162/ jocn.2007.19.12.1994

Potts, G. F., Martin, L. E., Burton, P., \& Montague, P. R. (2006). When things are better or worse than expected: The medial frontal cortex and the allocation of processing resources. Journal of Cognitive Neuroscience, 18, 1112-1119. doi: 10.1162/jocn.2006.18.7.1112

Ridderinkhof, K. R., Ullsperger, M., Crone, E. A., \& Nieuwenhuis, S. (2004). The role of the medial frontal cortex in cognitive control. Science, 306, 443-447. doi: 10.1126/science.1100301

Treisman, A. M., \& Gelade, G. (1980). Feature-integration theory of attention. Cognitive Psychology, 12, 97-136. doi: 10.1016/00100285(80)90005-5

Vidal, F., Hasbroucq, T., Grapperon, J., \& Bonnet, M. (2000). Is the "error negativity" specific to errors? Biological Psychology, 51, 109-128. doi: 10.1016/S0301-0511(99)00032-0

Yeung, N., Holroyd, C. B., \& Cohen, J. D. (2005). ERP correlates of feedback and reward processing in the presence and absence of response choice. Cerebral Cortex, 15, 535-544. doi: 10.1093/cercor/bhh153

Yeung, N., \& Sanfey, A. G. (2004). Independent coding of reward magnitude and valence in the human brain. Journal of Neuroscience, 24, 6258-6264. doi: 10.1523/Jneurosci.4537-03.2004

(ReceIVED November 6, 2013; ACCEPTED February 13, 2014) 